\title{
Owl monkey MHC-DRB exon 2 reveals high similarity with several HLA-DRB lineages
}

\author{
Carlos F. Suárez • Manuel E. Patarroyo • \\ Esperanza Trujillo $\cdot$ Mónica Estupiñán • \\ Juan E. Baquero $\cdot$ Carlos Parra $\cdot$ Raúl Rodriguez
}

Published online: 30 September 2006

(C) Springer-Verlag 2006

In the paper, we indicated the range of the Genbank codes as follows: A. nancymaae (Aona), A. nigriceps (Aoni) and A. vociferans (Aovo) MHC-DRB sequences were analysed

The online version of the original article can be found at http://dx.doi. org/10.1007/s00251-006-0127-0.

C. F. Suárez $\cdot$ M. E. Patarroyo $(\bowtie) \cdot$ E. Trujillo $\cdot$ M. Estupiñán

J. E. Baquero $\cdot$ C. Parra $\cdot$ R. Rodriguez

Fundación Instituto de Inmunmología de Colombia (FIDIC),

Carrera 50 No. 26-00,

Bogotá, Colombia

e-mail: mepatarr@fidic.org.co

M. E. Patarroyo

Universidad Nacional de Colombia,

Bogotá, Colombia
(GenBank accession numbers DQ162624-DQ162736 and AY563180-AY563263 respectively).

The correct information should read: A. nancymaae (Aona), A. Nigriceps (Aoni) and A. vociferans (Aovo) MHC-DRB sequences were analysed (GenBank accession numbers AY563180-AY563186, AY563188-AY563204, AY 563206-AY563234, AY563236-AY563237, AY5633239, AY563241-AY563263, DQ162624, DQ162626-DQ162630, DQ162633-DQ162635, DQ162645-DQ162648, DQ162660-DQ162661, DQ162668, DQ162676, DQ162679, DQ162682DQ162683, DQ162688, DQ162699, DQ162704DQ162705, DQ162710-DQ162711, DQ162718DQ162719, DQ162727, DQ162729, DQ162732, and DQ162736) 